\title{
M-ROAMi - Um Modelo para Promover o Desenvolvimento de Objetos de Aprendizagem Multimodais
}

\author{
Núbia dos S. R. S. dos Santos ${ }^{1}$, Leandro Krug Wives ${ }^{2}$, José Valdeni de Lima ${ }^{1,2}$, \\ Alexandre Hauber da Silva ${ }^{2,3}$ \\ ${ }^{1}$ PPGIE - Programa de Pós-Graduação em Informática na Educação- Universidade \\ Federal do Rio Grande do Sul (UFRGS) \\ Porto Alegre - RS - Brazil \\ ${ }^{2}$ PPGC- Programa de Pós-Graduação em Computação - Universidade Federal do Rio \\ Grande do Sul (UFRGS) \\ Porto Alegre - RS - Brazil \\ ${ }^{3}$ Technische Universität Kaiserslautern \\ Germany \\ nubia@sti.uff.br, \{valdeni, wives, ahsilva\}@inf.ufrgs.br
}

\begin{abstract}
The development of multimodal Learning Objects (LO) requires the inclusion of features in order to allow their use in a variety of ways and in different environments. In this context, this paper presents a model for the development and capacity building of LO based on QR Codes. Thus, the model proposes that any learning object stored in a Virtual Environment can be materialized (printed) with QR Codes and thus be used with the support of mobile devices, allowing mobility and more interactivity to the printed content. This article presents some applications that are needed to allow the use of proposed model and discusses some results of its use on existing LO and the students' satisfaction results when using these objects.
\end{abstract}

Resumo. A elaboração de Objetos de Aprendizagem multimodais (OAM) requer a sua capacitação no sentido de prover ou incluir elementos ou recursos que os permitam ser utilizados de vários modos, em diferentes ambientes. Nesse contexto, este artigo apresenta um modelo para o desenvolvimento e a capacitação multimodal de OAs utilizando QR Codes. Dessa forma, o modelo propõe que um objeto armazenado em um Ambiente Virtual possa ser materializado em mídia impressa e utilizado com o apoio de dispositivos móveis, permitindo mais mobilidade e interatividade ao conteúdo impresso. $O$ artigo apresenta os requisitos para elaboração de aplicações necessárias ao modelo e o resultado de satisfação dos alunos ao utilizá-lo.

\section{Introdução}

Um conteúdo educacional deve ser planejado visando as diferentes formas de utilização, uma vez que o aluno pode utilizar diferentes meios para acessar um conteúdo. $\mathrm{O}$ aluno 
pode utilizar um dispositivo móvel (smartphone, tablet, etc.), um computador, TV e inclusive mídia impressa. Quando o conteúdo é disponibilizado em diferentes formatos o aluno pode optar pela forma de utilizá-lo. No entanto, mesmo diante de tantos meios para acesso ao conteúdo digital, percebe-se que alguns preferem imprimir o conteúdo para estudo ao invés de utilizar meios digitais (Santos, Lima e Wives, 2012). Em pesquisa realizada por Santos, Lima e Wives (2012) sobre os meios de acesso ao conteúdo educacional, um dos motivos para impressão do conteúdo apontados pelos usuários quando perguntados sobre a preferência pelo estudo usando o papel está relacionado à mobilidade, ou seja, a facilidade de estudar em qualquer local sem ter com que se preocupar com questões como energia (luz), rede, etc. Os alunos também destacaram a facilidade de realizar anotações no material impresso, enquanto estudam o conteúdo. De acordo com pesquisas realizadas no Censo EaD.br 2010 (2012) em relação ao recurso principal de veiculação (meio-mestre) utilizado pelas instituições em seus cursos, de 198 instituições pesquisadas, 38 informaram meio impresso, 22 informaram internet, 23 informaram uso do CD-ROM e outras mídias e 115 não informaram. Ou seja, a maioria adotou o material impresso como principal recurso de mediação entre professores e alunos nos cursos oferecidos.

Nesse sentido, quando se elabora um conteúdo educacional seria relevante contemplar os diferentes meios nos quais ele será materializado, em especial a mídia impressa. O problema é que quando o material é disponibilizado em mídia impressa, existem muitas limitações, em especial porque ela se caracteriza pela pouca interatividade, servindo mais para a leitura do que para estudo do material (Belisário, 2006). Considerando tal limitação da mídia impressa, e o fato de ela ainda ser relevante para os alunos, este artigo propõe um modelo que capacita o conteúdo impresso de Objetos de Aprendizagem Multimodais (OA), através de códigos 2D, mais especificamente QR Codes ${ }^{1}$, agregando interatividade através de dispositivos móveis. Para Mayer e Moreno (2007) o modo é o código usado para representar a informação, isto é, verbal e não verbal, enquanto a modalidade é o sensor utilizado para receber a informação: auditivo e visual. Nesse sentido considera-se um OA Multimodal um objeto que permite ao usuário explorar os sentidos através de informação verbal e não verbal.

O modelo proposto neste artigo e alguns trabalhos relacionados são apresentados na seção 2. A seção 3 apresenta os requisitos para criação do aplicativo que integra o modelo, as aplicações desenvolvidas e os resultados da utilização do aplicativo pelos alunos. E, por fim, a seção 4 apresenta algumas considerações finais.

\section{Modelo M-ROAMi}

O modelo proposto inclui a utilização de mídia impressa em virtude de ainda ser muito utilizada na educação. Como forma de integração de todos os recursos para promover a mobilidade e interatividade do conteúdo educacional, a proposta apresenta alternativas para dar continuidade às atividades independente do meio e local utilizados. Dessa forma, a proposta é permitir que o OA disponibilizado no Ambiente Virtual de Aprendizagem (AVA) possa ser materializado em mídia impressa, de modo que os conteúdos dinâmicos possam ser representados por códigos 2D. O AVA utilizado para teste foi o Modular Object Oriented Developmental Learning Environment (Moodle),

\footnotetext{
${ }^{1}$ QR Code é marca registrada da DENSO WAVE INCORPORATED.
} 
um Sistema Open Source de Gerenciamento de Cursos, utilizado como plataforma oficial para desenvolvimento de cursos on-line e/ou como plataforma para comunicação e troca de material entre professores e alunos no ensino presencial (Moodle, 2010). O Moodle é um dos AVAs mais utilizados no ensino a distância, bem como no ensino presencial servindo de complemento, no gerenciamento de conteúdo, organização das aulas, realização de atividades, etc. O Moodle oferece diversas possibilidades de atividades e recursos, inclusive o armazenamento de OAs que possuem recursos do próprio Moodle. Alguns dos recursos do Moodle que podem ser acessados através de um OA são: fórum, página Web, vídeos, áudio, atividades, questionários, entre outros. Além das possibilidades fornecidas pelos AVAs, pode-se citar o M-learning. Conforme definido em Batista, Behar e Passerino (2012), o M-learning é o campo de pesquisa que investiga como os dispositivos móveis podem contribuir para a aprendizagem. Vale ressaltar que o desenvolvimento de conteúdos educacionais para dispositivos móveis oferece benefícios relacionados à mobilidade, ubiquidade e interatividade.

No entanto, dispositivos móveis apresentam algumas limitações, tais como dificuldade de leitura e escrita de acordo com resolução de tela, duração de bateria, instabilidade na rede, entre outras. Um recurso que pode ser utilizado para integrar o meio físico ao digital e ainda ser utilizado por dispositivos móveis são os códigos 2D, códigos de duas dimensões. O código 2D utilizado para o modelo foi o QR Code, código criado pela empresa japonesa Denso Wave em 1994, é um código bidimensional que permite o carregamento de informações na direção vertical, bem como na horizontal. O QR Code pode ser lido em dispositivos móveis (celular com câmera), tablet, computador e, uma vez acessado, permite realizar ações tais como ler um texto, acessar um site, realizar ligação, enviar mensagens de texto, entre outras. Os QR Codes estão sendo muito utilizados em todos os lugares para diversas finalidades, inclusive na educação (Educause, 2009; Ramsden, 2008). Códigos como o QR Code permitem a convergência absoluta de mídias de informação, pois conectam documentos estáticos à Internet. Nesse contexto, percebe-se a existência de vários recursos que podem ser integrados para oferecer mais possibilidades de acesso e continuidade ao ensino. Dessa forma, o modelo proposto, Modelo de representação de OAs Multimodais e Interativos (M-ROAMi), contempla a integração de recursos de modo a permitir a materialização do OA Multimodal (armazenado no AVA) em mídia impressa usando a tecnologia QR Code para acesso via dispositivo móvel através de um aplicativo Android (seção 3). Dessa forma, o modelo pode ser utilizado no ensino à distância e no ensino presencial. O modelo é composto de metadados de descrição dos recursos de forma a possibilitar a criação de regras para facilitar a materialização dos recursos do OA. O modelo integra os metadados do modelo UMBRELO (Santos et al., 2012), o qual inclui metadados do padrão LOM e oferece uma estrutura que descreve de forma detalhada os conteúdos (recursos) de OAs. Dessa forma, utilizou-se o modelo UMBRELO, incluindo os metadados de vídeo, áudio, animação e questionários. Dentre alguns exemplos de trabalhos realizados com dispositivos móveis, mais especificamente na plataforma Android, pode-se citar o software Labirinto do Rato, voltado para o desenvolvimento do raciocínio lógico infantil (Silva, Nóbrega e Jacob, 2011). A estratégia é utilizar o software como ferramenta educacional para os alunos que apresentam dificuldades de concentração. Outras aplicações voltadas para a realização de atividades educacionais com dispositivos móveis são apresentadas por Orlandi e Isotani (2012), com o desenvolvimento de um sistema de autoria e distribuição de conteúdo educacional interativo para dispositivos móveis (smartphones e tablets) e Silva e Rebouças (2011), 
apresentando o projeto do jogo MyQuimica, que objetiva auxiliar no ensinoaprendizagem de química, tendo como foco as nomenclaturas químicas. Fernandes et al.(2012) apresentam uma proposta similar de integração de dispositivos móveis com o Moodle para aplicação de questionários, porém não foi mencionado no trabalho o uso de QR Codes em mídia impressa (materialização do OA).

\section{Aplicações desenvolvidas para integração ao modelo}

Em atendimento aos objetivos do modelo, descrito na seção 2, foram desenvolvidas aplicações para realizar a materialização em mídia impressa, registrar as informações (representadas por QR Codes) nos dispositivos móveis e atualizar as respostas (registros) no AVA, conforme apresenta a arquitetura (Figura 1).

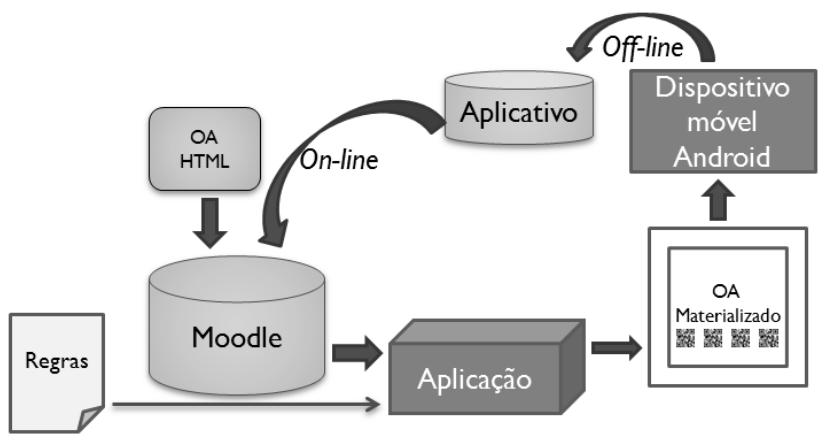

Figura 1. Arquitetura proposta

Dentre as opções de recursos do $\mathrm{OA}$, este artigo destaca os questionários para realização de um teste inicial do modelo proposto. Deve-se considerar a existência de diferentes configurações para o recurso questionário. No Moodle tal recurso pode conter questões de múltipla escolha, verdadeiro/falso, discursiva, entre outras. Uma aplicação foi desenvolvida em PHP para gerar o conteúdo, nesse caso o questionário, materializado com QR Codes (Figura 2). Ao acessar a aplicação o usuário informa o login e senha do Moodle. A aplicação exibe todos os questionários criados pelo usuário, com as opções de detalhar e gerar QR Codes. Ao selecionar um questionário e clicar na opção Gerar QR Code, um documento em formato PDF é gerado. Tal documento é a materialização do questionário (com QR Codes) e poderá ser utilizado com o aplicativo Android (seção 3.1).

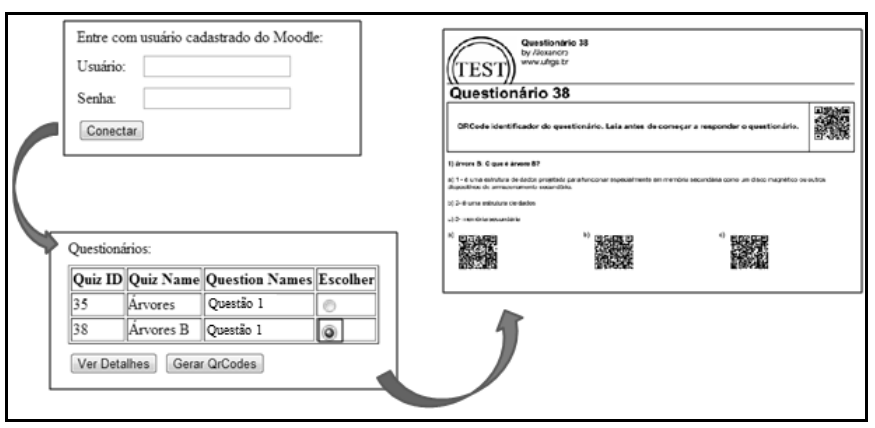

Figura 2. Aplicação para materialização do conteúdo 
Ultimamente, muitos aplicativos com objetivos educacionais têm sido desenvolvidos para dispositivos móveis, inclusive Android. Conforme aponta Fernandes et al. (2012), no Brasil, 28\% dos celulares ativos utilizam o Android enquanto o principal concorrente, o iOS, é utilizado em $10 \%$ dos aparelhos. O Android é um esforço da Google em colaboração com a Open Handset Alliance. É uma plataforma de software de aplicativos para dispositivos móveis (celulares) com código-fonte aberto, que inclui sistema operacional baseado em Kernel Linux, aplicativos de usuário, bibliotecas de código, frameworks de aplicativo, suporte a multimídia entre outros (King, Ableson e Sen, 2012).

Considerando o aumento de uso pelos usuários e a vantagem de possuir código aberto, a proposta integra o desenvolvimento de um aplicativo usando o sistema Android (seção 3.1).

\subsection{Requisitos para criação de aplicativos}

Para desenvolvimento do aplicativo Android e da aplicação que gera o conteúdo materializado, alguns requisitos foram elencados, conforme apresenta o Quadro 1. Vale ressaltar que foi utilizado o recurso questionários do Moodle para o teste inicial do modelo.

\section{Quadro 1. Requisitos para aplicativo Android}

\begin{tabular}{|l|l|}
\hline \multicolumn{1}{|c|}{ Requisitos } & \multicolumn{1}{c|}{ Descrição } \\
\hline $\begin{array}{l}\text { Visualizar } \\
\text { questionários do } \\
\text { Moodle }\end{array}$ & $\begin{array}{l}\text { O professor pode visualizar todos os questionários do Moodle e } \\
\text { selecionar o questionário para materialização. }\end{array}$ \\
\hline Gerar documento & $\begin{array}{l}\text { O professor pode gerar um questionário materializado, em formato } \\
\text { PDF, com QR Codes. }\end{array}$ \\
\hline $\begin{array}{l}\text { Realizar cadastro no } \\
\text { aplicativo Android }\end{array}$ & $\begin{array}{l}\text { O aluno pode realizar o cadastro no aplicativo para responder o } \\
\text { questionário. }\end{array}$ \\
\hline $\begin{array}{l}\text { Logar no aplicativo } \\
\text { Android }\end{array}$ & $\begin{array}{l}\text { O aluno pode efetuar login, uma vez cadastrado, para utilizar o } \\
\text { aplicativo. }\end{array}$ \\
\hline $\begin{array}{l}\text { Informar questionário a } \\
\text { ser respondido }\end{array}$ & $\begin{array}{l}\text { O aluno pode informar o questionário a ser respondido para iniciar } \\
\text { a atividade. }\end{array}$ \\
\hline $\begin{array}{l}\text { Registrar resposta para } \\
\text { questão }\end{array}$ & $\begin{array}{l}\text { O aluno pode registrar as respostas das questões no aplicativo de } \\
\text { forma off-line. }\end{array}$ \\
\hline $\begin{array}{l}\text { Alterar opção de } \\
\text { resposta }\end{array}$ & $\begin{array}{l}\text { O aluno pode alterar a opção registrada no aplicativo, desde que } \\
\text { não tenha atualizado o questionário no Moodle. }\end{array}$ \\
\hline Detalhar questionário & $\begin{array}{l}\text { O aluno pode detalhar o questionário para verificar o histórico das } \\
\text { questões respondidas. }\end{array}$ \\
\hline Atualizar questionário & $\begin{array}{l}\text { O aluno pode atualizar (enviar) o questionário para registro de suas } \\
\text { respostas no Moodle. }\end{array}$ \\
\hline Fornecer feedback & $\begin{array}{l}\text { O aluno pode obter feedback do questionário respondido no } \\
\text { aplicativo e/ou no Moodle. }\end{array}$ \\
\hline
\end{tabular}


Para realizar a integração do aplicativo Android com o Moodle foram utilizadas as tabelas:

- mdl_user: para validar o login do usuário; logado;

- mdl question: para buscar as questões criadas e/ou modificadas pelo usuário

- mdl_quiz_question_instances: para associar as questões aos questionários (quizes);

- mdl_quiz: para buscar as informações dos questionários;

- mdl_question_answers: para buscar as respostas de cada questão, armazenadas em uma tabela separada de $m d l \_q u e s t i o n$.

Ao instalar o aplicativo no dispositivo móvel o usuário, nesse caso o aluno, deve realizar o cadastro informando os dados de login no Moodle. A validação do usuário no Moodle ocorre somente no processo de atualização (envio) do questionário no Moodle. Após realizar o login o aplicativo apresenta uma tela com três abas e orientações sobre os recursos do aplicativo, conforme apresenta a Figura 3.

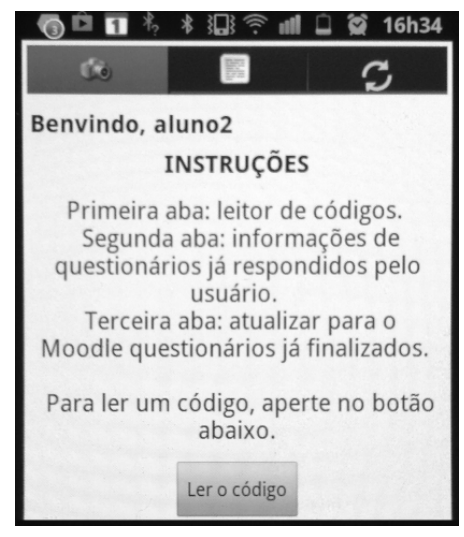

Figura 3. Tela inicial do aplicativo Android

Para iniciar o questionário o aluno deve clicar na opção "ler o código" e direcionar a câmera para o QR Code que representa o questionário a ser iniciado. Em seguida, o aluno poderá decodificar as questões do questionário. $\mathrm{O}$ aluno deve escolher uma das opções de resposta da questão direcionando a câmera para o QR Code que representa a opção que julgar correta. $\mathrm{O}$ aplicativo apresenta o resumo da leitura, ou seja, a questão e a resposta registrada. O aplicativo registra todas as questões respondidas e os detalhes podem ser visualizados na segunda aba do aplicativo. O aluno pode responder as questões de forma não sequencial e alterar as respostas registradas até o momento de atualização (envio) do questionário ao Moodle.

Após responder todas as questões do questionário o aluno pode atualizar as questões no Moodle. Para isso, deve acessar a $3^{\mathrm{a}}$ aba, escolher o questionário, visualizar os dados e clicar em Atualizar (Figura 4). Para atualizar o questionário no Moodle é necessário conexão com a Internet para validação do usuário no Moodle. Mas o aluno pode visualizar o resultado do questionário (feedback) diretamente no aplicativo de forma off-line. 


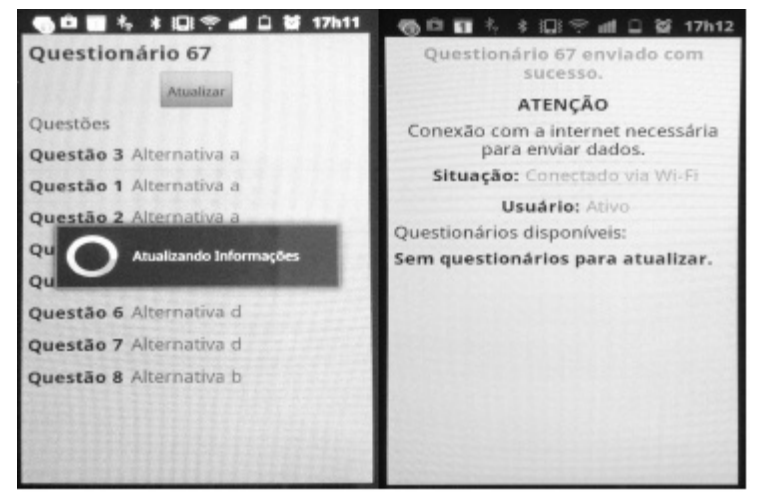

Figura 4. Tela de atualização do questionário no Moodle e tela de confirmação de envio, respectivamente

Ao finalizar o processo de atualização (envio), o dispositivo apresenta a confirmação do envio (Figura 4 à direita). Então, ao acessar o Moodle o aluno visualizará o questionário finalizado com o resultado e o detalhamento da avaliação. $\mathrm{O}$ aplicativo foi desenvolvido priorizando a simplicidade para permitir que os alunos tenham facilidade ao utilizá-lo. Todos os requisitos elencados foram implementados para o teste inicial com questionários. $\mathrm{O}$ aplicativo e o conteúdo materializado foram utilizados em sala de aula com alunos para efeitos de validação e obtenção de feedback em relação à usabilidade.

\subsection{Avaliação dos aplicativos}

O questionário materializado e o aplicativo Android foram utilizados em três turmas: duas turmas de cursos de graduação na Universidade Federal do Rio Grande do Sul e uma turma de ensino médio, na modalidade Educação de Jovens e Adultos (EJA). Devido às especificações necessárias para uso do aplicativo, apenas alguns alunos puderam participar do experimento. No total, 14 alunos utilizaram o aplicativo para responder o questionário sobre o conteúdo abordado pelo OA. O OA, desenvolvido em HTML 5, armazenado no Moodle e materializado em mídia impressa com QR Code, abordou o conteúdo Segurança na Web, apresentando os riscos existentes, o conceito de Engenharia Social e medidas de prevenção na Web. Os recursos dinâmicos do OA tais como vídeo, áudio, animação, atividades, dicas, entre outros, foram representados por QR Codes no OA materializado. Os alunos que utilizaram o OA materializado e responderam o questionário (sobre Segurança na Web) gerado pela aplicação (Figura 2), usando o aplicativo Android, responderam o questionário de avaliação (Tabela 1) do modelo. Para elaboração do questionário considerou-se algumas das heurísticas de usabilidade (Nielsen, 1994). O questionário de avaliação do modelo foi elaborado usando escala Likert $^{2}$ de 5 pontos, sendo eles: S (Sim), P+ (Parcialmente com poucas restrições), P (Parcialmente), P- (Parcialmente com muitas restrições) e N (Não) e os respectivos valores $1.00,0.75,0.50,0.25,0$, conforme apresenta a Tabela 1 . O resultado obtido com o uso do modelo evidencia a predominância de respostas positivas (entre $\mathrm{S}$ e P), ou seja, um indicativo de satisfação em relação ao modelo proposto.

\footnotetext{
${ }^{2}$ Escala Likert- é uma escala utilizada em questionários para medir o grau de acordo ou desacordo em uma declaração.
} 
Tabela 1. Resultado do questionário sobre o aplicativo Android

\begin{tabular}{|c|c|c|c|c|c|}
\hline Questões & S & $\mathrm{P}+$ & $\mathrm{P}$ & P- & $\mathrm{N}$ \\
\hline 1-O aplicativo funciona corretamente. & 9 & 3 & 2 & & \\
\hline 2- O aplicativo é fácil de utilizar. & 5 & 7 & 2 & & \\
\hline 3- $\mathrm{O}$ aplicativo permite mais interatividade ao questionário impresso. & 9 & 3 & 2 & & \\
\hline 4- O questionário materializado com QR Code é fácil de utilizar. & 6 & 4 & 3 & 1 & \\
\hline 5- $\mathrm{O}$ aplicativo registra corretamente as questões respondidas. & 12 & & 2 & & \\
\hline 6- As informações do aplicativo são suficientes para o entender o funcionamento. & 4 & 5 & 5 & & \\
\hline 7-O aplicativo permite a atualização do questionário no Moodle. & 10 & 2 & 2 & & \\
\hline 8- O aplicativo fornece feedback apropriado sobre a ação do usuário. & 7 & 3 & 4 & & \\
\hline 9- As formas de realização de ações semelhantes são consistentes. & 9 & 3 & 2 & & \\
\hline 10 - As informações apresentadas no aplicativo são relevantes. & 8 & 3 & 3 & & \\
\hline 11- A possibilidade de utilizar o aplicativo off-line auxilia na realização de atividades. & 13 & & 1 & & \\
\hline 12 - O aplicativo oferece mais mobilidade para realizar tarefas. & 9 & 3 & 2 & & \\
\hline 13 - O questionário impresso favorece a reflexão e a concentração para respondê-lo. & 8 & 2 & 4 & & \\
\hline 14 - A disposição de menus e recursos favorece a utilização do aplicativo. & 9 & 2 & 2 & 1 & \\
\hline 15 - A utilização do aplicativo facilita a realização de atividades. & 7 & 4 & 3 & & \\
\hline 16- O guia de uso do aplicativo auxilia no entendimento de suas funcionalidades. & 12 & 1 & 1 & & \\
\hline 17- É necessário aprender muitos conceitos para saber utilizar o aplicativo. & 2 & 1 & 4 & 1 & 6 \\
\hline 18- $\mathrm{O}$ aplicativo fornece segurança ao usuário das ações realizadas. & 7 & 4 & 3 & & \\
\hline $\begin{array}{l}\text { 19- O aplicativo pode ser usado facilmente por qualquer usuário, pois não exige } \\
\text { conhecimentos técnicos. }\end{array}$ & 7 & 3 & 4 & & \\
\hline
\end{tabular}

Conforme apresentado na Tabela 1 a resposta $\mathrm{S}(\mathrm{Sim})$ para questões afirmativas prevaleceu sobre as demais opções de respostas relacionadas à satisfação, representando $58 \%$ das respostas. Os dados da Tabela 1 representam as frequências das respostas dos alunos para cada questão. Vale ressaltar que a questão 17 é invertida, pois a resposta ' $N$ ' favorece para um resultado positivo. Além do questionário para avaliação do aplicativo Android apresentado na Tabela 1, os alunos relataram a experiência:

- "A experiência foi muito interessante, pois ao mesmo tempo que o aluno lê o material, pode entrar em contato com o conteúdo interativo."

- "Acho que foi uma grande experiência ao ter contato com essa nova tecnologia, e uma tecnologia que vem sendo cada vez mais utilizada."

- "Tanto o conteúdo como as diversas atividades que o material possuía possibilitou uma aprendizagem mais dinâmica, fugindo um pouco do "velho padrão"."

- "Seria uma boa alternativa para estudo, pois poderia ser usado em qualquer lugar com dispositivos portáteis." 
Diante do resultado obtido, percebe-se que a integração desses recursos pode oferecer novas possibilidades de uso do conteúdo educacional, considerando as vantagens inerentes de cada meio. O resultado também evidenciou algumas melhorias necessárias no que tange às informações disponíveis no $\mathrm{OA}$ materializado e no aplicativo, de modo a facilitar o entendimento das funcionalidades e do processo. $\mathrm{O}$ teste inicial foi realizado com questionários, porém regras, baseadas nos metadados do modelo, estão em desenvolvimento para permitir a materialização de outros recursos, além de questionários. Tais recursos representados por QR Codes integrarão o OAM, permitindo o registro de ações dos alunos mesmo quando utilizam o material impresso, seguindo o modelo apresentado nesse artigo.

\section{Considerações finais}

O artigo apresentou um modelo que objetiva proporcionar novas possibilidades de acesso aos conteúdos educacionais com a inclusão de recursos de tecnologia de códigos 2D em OAM materializados em mídia impressa. Dessa forma, a materialização de OAs em mídia impressa com a utilização de QR Codes e uso de dispositivos móveis parece ser uma alternativa para reduzir as limitações inerentes a cada meio. Conforme aborda Masetto (2007) 'não podemos ter a esperança de que uma ou duas técnicas, repetidas à exaustão, deem conta de incentivar e encaminhar toda a aprendizagem esperada" (Masetto, 2007, p.143). Percebe-se que uma tecnologia apenas não é suficiente para permitir a continuidade do ensino, pois todas possuem limitações. As aplicações desenvolvidas para materialização e registro das ações dos alunos no dispositivo foram testadas inicialmente com questionários. $\mathrm{O}$ modelo foi testado com alunos de cursos de graduação e ensino médio para validação das aplicações e apresentou resultados satisfatórios. Algumas dificuldades encontradas no desenvolvimento do aplicativo estão relacionadas à complexidade do modelo e a documentação insuficiente. Uma das contribuições do modelo é permitir que o professor possa utilizá-lo para criar OAM, gerando questionários materializados de forma automática. Ou seja, usando as aplicações desenvolvidas, o professor pode materializar os questionários criados no Moodle e disponibilizá-los para que os alunos respondam usando o aplicativo Android. Tal OAM pode ser aplicado no ensino presencial ou a distância, como uma alternativa para os alunos que utilizam a mídia impressa para estudo. $\mathrm{O}$ modelo atende, no momento, aos questionários, mas a proposta integra a materialização de todos os conteúdos de um OAM. Regras, baseadas nos metadados do modelo, estão sendo elaboradas para permitir a materialização dos demais recursos de um OA. Ou seja, os recursos dinâmicos dos OAM serão representados por QR Codes automaticamente através de uma ferramenta, de acordo com as regras estabelecidas.

\section{Agradecimento}

Agradecimento à Prof ${ }^{a}$ Clevi Elena Rapkiewicz pela possibilidade de realização da Oficina sobre códigos 2D na turma de Educação de Jovens e Adultos (EJA). À SEAD/UFRGS pelo apoio financeiro, propiciando o desenvolvimento dos Objetos de Aprendizagem e do aplicativo.

\section{Referências}

Batista, S.C.F.; Behar, P.A.; Passerino, L.M. (2012), M-learnMat: Modelo Pedagógico para Atividades de M-learning em Matemática, Anais do XXIII SBIE-Simpósio 
Brasileiro de Informática na Educação, Concurso de Teses e Dissertações em Informática na Educação (CTD-IE 2012), Rio de Janeiro.

Belisário, A. (2006) O material didático na educação a distância e a constituição depropostas interativas, In: SILVA, M. (org), Educação Online, São Paulo:Loyola, p. 137-148

Censo, Ead.br (2012), Relatório analítico da aprendizagem a distância no Brasil 2010, São Paulo: Pearson Education do Brasil.

Educause, (2009) Educause Learning Initiative, 7 things you should know about...QR Codes, Disponível em: http://net.educause.edu/ir/library/pdf/ELI7046.pdf, Acesso em: Jan. 2011.

Fernandes, K. T. et al, (2012) Question Mobile: Ampliando Estratégias de Avaliação da Aprendizagem por Meio de Dispositivos Móveis, Anais do XXIII SBIE - Simpósio Brasileiro de Informática na Educação, Rio de Janeiro.

King, C.; Ableson, W.F.; Sen, R.(2012) Android em ação, Elsevier-Campus.

Masetto, M. T. (2007) Mediação pedagógica e o uso da tecnologia, In: Novas tecnologias e mediação pedagógica, Moran, J.M., Masetto, M.T., Behrens,M.A., SP:Papirus, $13^{\mathrm{a}}$ ed.

Mayer, R.E., Moreno, R. (2003) Nine Ways to Reduce Cognitive Load in Multimedia Learning Educational Psychologist, 38(1), 43-52

Moodle, Moodle, 2010. Disponível em http://Moodle.org/about/ Acesso em: 10 fev.2011.

Nielsen, J., (1994) Heuristic Evaluation. In Nielsen, J., and Mack, R.L. (Eds.), Usability Inspection Methods, New York: John Wiley \& Sons.

Orlandi, B.H.; Isotani, S. (2012) Uma Ferramenta para Distribuição de Conteúdo Educacional Interativo em Dispositivos Móveis, Anais do XXIII SBIE - Simpósio Brasileiro de Informática na Educação, Rio de Janeiro.

Ramsden, A. (2008) The use of QR Codes in Education: a getting started guide for academics. Online Publications Store, University of Bath Opus. Disponível em: $<$ http://opus.bath.ac.uk/> Acesso em: 14 set. 2010.

Santos, N.S.R.S.; Lima, J.V.; Wives, L.K.; (2012) Integração de recursos para acesso aos Objetos de Aprendizagem Multimodais, Renote-Novas Tecnologias na Educação. V.10 N3.

Santos, N.S.R.S et al (2012), Metadados para Objetos de Aprendizagem: prova de conceito do modelo UMBRELO, Anais do XXIII SBIE - Simpósio Brasileiro de Informática na Educação, Rio de Janeiro

Silva, D.L.M, Rebouças, A.D.D.S. (2011) MyQuímica - Um jogo para auxiliar no ensino aprendizagem das nomenclaturas químicas, Anais do XXII SBIE-Simpósio Brasileiro de Informática na Educação, Aracaju.

Silva, S.S.R., Nobrega, S.M., Jacob Jr. A.F.L. (2011) Labirinto do Rato: jogo educacional infantil para dispositivos móveis, Anais do XXII SBIE - Simpósio Brasileiro de Informática na Educação, Aracaju. 